\title{
REFLEXIONES ACERCA DE LA TEORÍA DE RICARDO SOBRE LA GANANCIA Y LA TEORÍA DE MARX SOBRE LA PLUSVALIA *
}

\author{
Carlos Roces \\ El Colegio de México
}

\section{INTRODUCCIÓN}

EN ESTE BREVE ensayo se intenta explicar algunas diferencias importantes entre la teoría de Marx sobre la plusvalía y la concepción de Smith y, fundamentalmente, de Ricardo acerca de la ganancia. El tema nos lleva en forma directa a la relación entre trabajo y valor en un sistema de producción basado en trabajo asalariado y capital.

Debo adelantar que sólo examinaré en detalle lo que Ricardo considera como la ganancia derivada del capital-salarios. Por consiguiente, al tratar de Marx, me circunscribiré al problema de la plusvalía. Puesto que dejaré de lado la concepción ricardiana de la ganancia emanada del capital fijo y de distintos períodos de producción, no tengo por qué referirme a la teoría de la ganancia de Marx.

Las conclusiones a que llego podrían resumirse así:

Smith y Ricardo postulan que el trabajo contenido en el salario es menor al trabajo que el obrero vende al capitalista, pero ninguno de los dos autores acierta a enmarcar esta conclusión dentro de una teoría del valor.

Marx logra hacerlo con la teoría de la plusvalía. La clave del hallazgo está en su concepción del valor. El valor aparece claramente como la categoría que expresa la relación de producción dominante de todo modo de producción con vistas al cambio. Concebido el capitalismo como modo de producción para el cambio, la propia categoría valor y las características que diferencian a este sistema de la producción mercantil simple son los elementos que ayudan a definir la mercancía vendida por el obrero al capitalista y, por este medio, a resolver el dilema que formulan Smith y Ricardo.

* Agradezco a mis colegas de El Colegio de México que hayan discutido conmigo una versión preliminar de este trabajo y al Sr. Wenceslao Roces que haya revisado la redacción de la versión final. 


\section{LA TEORÍA RICARDIANA DE LA GANANCIA}

Desde el "Ensayo sobre las utilidades" 1 y, claramente, en los Principios $^{2}$ Ricardo señala que el valor de cambio de cualquier mercancía frente a la mercancía dinero depende de la cantidad de trabajo empleada en producirlas - en cada caso conforme a la productividad del trabajo de la dosis final de capital- y del tiempo transcurrido hasta la realización total de la ganancia del capital invertido en la producción de una y otra.

El valor-dinero de cualquier mercancía es, deducida la renta, el total del capital invertido en producirla más la ganancia. Capital es la suma de salarios, materias primas, desgaste de bienes de producción durables y ganancias no realizadas en el mismo lapso de tiempo que las obtenidas en la producción de la mercancía dinero. Cada uno de estos elementos del capital rinde una ganancia de acuerdo con la tasa normal. Los salarios y la ganancia correspondiente son, totalmente, expresión en dinero de la cantidad de trabajo empleado de manera directa en producir la mercancía. De todo el capital y la ganancia restantes sólo representan trabajo los salarios y la ganancia correspondiente a la mano de obra empleada directamente en la producción de las materias primas y de los bienes de producción. El resto no implica trabajo. Es ganancia acumulada como capital por no haberse realizado en el mismo período que la de la mercancía dinero. A su vez, este capital engendra ganancia.

Si cambia el salario-dinero, porque varíe la cantidad de trabajo empleado en producir las mercancías-salario, ninguna mercancía que siga produciéndose con la misma cantidad de trabajo sufre cambio alguno en la parte de su valor-dinero que representa el capital salarios más la ganancia correspondiente. Este total permanece inalterado porque la ganancia varía en sentido opuesto y en la misma proporción que el capital. Sólo cambia la parte del valor-dinero que no representa trabajo. Al variar la tasa de ganancia del capital-salarios varía la tasa de ganancia del resto del capital. Es decir, toda ganancia no realizada se calcula a la nueva tasa que marque el capital-salarios. Y, su vez, como capital, arroja una ganancia bajo la nueva tasa.

Con base en esta relación entre salario y valor-dinero de las mercancías, Ricardo criticó la tesis de Smith de que el valor de todas las mercancías se modifica en el mismo sentido que el salario. Esto sólo ocurre con las mercancías en que la proporción de capital y ganancia que representa trabajo, frente a capital y ganancia que no representa trabajo, sea mayor que la proporción correspondiente en la mercancía-dinero. Para

1 "Un ensayo acerca de la influencia del bajo precio del trigo en las ganancias del capital", Obras de Ricardo, México, Fondo de Cultura Económica, 1960, t. IV.

2 Principios de economía política y tributación, México, Fondo de Cultura Económica, 1959. 
aquellas en que la proporción sea menor, el cambio en el valor-dinero es opuesto al cambio en el salario.

En todo este análisis, es fundamental la relación entre capital-salarios y ganancia de dicho capital. ¿Por qué el cambio del salario-dinero no afecta a la suma de capital-salarios y su ganancia, sino, únicamente, a la distribución de ese total? ¿Por qué se da una relación inversa entre el salario y la ganancia derivada de él?

Cuando Ricardo analiza la ganancia global ${ }^{3}$ toma el fondo de salarios como único capital invertido en la producción de cualquier mercancía. Al descartar el capital que representan las ganancias no realizadas en el mismo período que las de la mercancía dinero, el autor supone, de acuerdo con lo señalado en párrafos anteriores, que el valor-dinero de las mercancías depende por entero de la cantidad de trabajo empleado en producirlas con respecto a la cantidad de trabajo que la mercancía dinero requiere. Cuando todo el capital se reduce a salarios el trabajo es el único determinante del valor de cambio.

Mediante un esquema algebraico sencillo es posible reproducir fielmente el planteamiento ricardiano en torno a la ganancia. Supongamos tres mercancías: $A, B$ y dinero $D$. La producción de cada una se incrementa al aplicar dosis sucesivas de capital-salarios, o sea, dosis de trabajo directo.

En $A$ y $D$ el producto medio por hora-hombre no varía de una dosis a otra y en $B$ el producto medio decrece con cada dosis adicional. Es decir, en $A$ y $D$ el producto medio de la dosis final es igual que el de las dosis intermedias y en $B$ es menor.

Llamemos $a, b$ y $d$ al producto medio de las dosis finales de capital empleadas en la producción de $A, B$ y $D$. Según la formulación de Ricardo, el valor-trabajo por unidad de cada mercancía es:

$\frac{1}{a}, \frac{1}{b}$ y $\frac{1}{d}$. Por tanto, el valor-dinero de $A$ y $B$ es: $\frac{d}{a}$ y $\frac{d}{b}$.

El salario real por hora-hombre es una cantidad dada de $B, b^{*}$, independientemente de la productividad del trabajo en cualquiera de las tres ramas o del monto total de capital empleado en cada una. El valor-dinero del salario es: $\frac{d}{b} b^{*}$.

En $A$ y $D$ la ganancia por hora-hombre, valuada en dinero, es:

$$
\begin{aligned}
& \frac{d}{a} a-\frac{d}{b} b^{*}=d\left(1-\frac{b^{*}}{b}\right) ; d-\frac{d}{b} b^{*}=d\left(1-\frac{b^{*}}{b}\right) \\
& \quad 3 \text { op. cit., Caps. V y VI. }
\end{aligned}
$$


En $B$ la ganancia por hombre empleado con la dosis final de capital es:

$$
\frac{d}{b} b-\frac{d}{b} b^{*}=d\left(1-\frac{b^{*}}{b}\right)
$$

La ganancia por hora-hombre correspondiente a cualquier dosis intermedia es la misma, puesto que la renta de la tierra absorbe la diferencia entre el valor-dinero de $B$ calculado a partir del producto medio del trabajo de la dosis final y el valor-dinero calculado con base en el producto medio de la dosis intermedia. Es decir:

$$
R=\frac{d}{b} b^{\prime}-\frac{d}{b^{\prime}} b^{\prime}=d\left(\frac{b^{\prime}}{b}-1\right)
$$

en donde: $R=$ Renta por hora-hombre; $b^{\prime}=$ Producto por hora-hombre, dentro de la dosis intermedia de capital.

Es decir, la ganancia por hora-hombre empleada con la dosis intermedia es:

$$
\frac{d}{b} b^{\prime}-d\left(\frac{b^{\prime}}{b}-1\right)-\frac{d}{b} b^{*}=d\left(1-\frac{b^{*}}{b}\right)
$$

Al cambiar el valor monetario del salario, porque varía la productividad del trabajo empleado en producir $B$, no cambia el valor monetario de $A$ y $D$ ya que $a$ y $d$ no dependen de $b$. Cambia, en sentido opuesto al salario, la ganancia por hora-hombre empleada en las tres ramas.

En este esquema, la distribución del producto en salarios y ganancias no afecta al valor-dinero de las mercancías, puesto que no influye en la cantidad de trabajo empleada en producirlas. Por el contrario, el valordinero de las mercancías-salario sí afecta a esta distribución.

En cambio, como hemos señalado antes, la distribución y el valordinero de las mercancías se influyen mutuamente cuando éste se determina por la cantidad de trabajo y las ganancias que, no realizadas, se acumulen como capital.

A partir de la premisa de que el único capital son los salarios, ¿cómo explica Ricardo la ganancia? ¿Por qué motivo, si una hora-hombre produce $b$ o $b^{\prime}$ recibe una cantidad menor, $b^{*}$ ? o, si consideramos las otras mercancías, ¿por qué una hora-hombre no percibe como salario el equivalente a una hora-hombre, sino $\frac{1}{b} b^{*}-$ menor que 1 siempre que $b$ sea
mayor que $b^{*}$ ?

En La riqueza de las naciones ${ }^{4}$ Adam Smith señala con claridad que,

4 Investigación sobre la naturaleza y las causas de la riqueza de las naciones, México, Fondo de Cultura Económica, 1958. 
al acumularse capital en manos de ciertos individuos, éstos ponen a trabajar a personas con capacidad de producción, suministrándoles materias primas y medios de vida, con el fin de obtener una ganancia de lo que el trabajador añade al valor de las materias primas. El valor añadido se desdobla en dos partes: salarios y ganancias por el capital adelantado en salarios y materias primas.

El trabajador sólo puede añadir trabajo. El trabajo incorporado por él a las materias primas se divide en dos partes. Una es el salario y la otra es trabajo que el capitalista vende sin haberlo comprado, al vender la mercancía para cuya producción adelantó capital.

Si el capitalista vende la mercancía por el equivalente de la cantidad de trabajo que ésta encierra, es decir, si la cambia por una mercancía que contenga la misma cantidad de trabajo, la ganancia se obtiene por vender, sin haberla pagado, una parte del trabajo cristalizado en la mercancía.

En esta formulación de Smith la ganancia es trabajo no retribuido. ¿Cómo es posible tal apropiación? El autor no explica por qué las condiciones de trabajo pertenecen a una clase ni la facultad de vender fuerza de trabajo a otra. Parte como de algo dado de esta separación, que es la premisa necesaria de lo que él mismo percibe como trabajo no retribuido.

Según Smith, cuando los productores son propietarios de las mercancías que producen $\mathrm{y}$, por lo tanto, pueden venderlas, el valor se halla determinado por la cantidad de trabajo contenido en ellas. Una mercancía producida en $x$ horas-hombre podrá venderse por otra que encierra $x$ horas-hombres. Como la cantidad de trabajo que puede comprarse con una mercancía es igual a la cantidad de trabajo que ésta contiene, ambas magnitudes son la medida de su valor. Es decir, en términos del autor, el valor del trabajo es el valor del producto del trabajo.

Cuando el producto no pertenece al trabajador, el valor del trabajo es menor que el valor del producto de él, puesto que hay trabajo no retribuido. En estas condiciones, al enfrentarse diversos poseedores de mercancías, cada uno compra tanto trabajo cristalizado en cualquier mercancía como trabajo contenga lo que vende, pero al enfrentarse el trabajador y el capitalista, el primero vende más trabajo vivo del que compra, cristalizado en las mercancías-salario. Es decir, trabajo comprado y trabajo contenido son lo mismo cuando se enfrentan mercancías como cristalización de trabajo, y no son lo mismo cuando se enfrentan trabajo vivo y trabajo cristalizado en mercancías-salario.

Sin embargo, el trabajo vivo, para Smith, es una mercancía. El obrero vende trabajo vivo al capitalista. Además, si las mercancías que son cristalización de trabajo compran la misma cantidad de trabajo que contienen es porque en sus distintas fases de producción se ha empleado trabajo vivo. En este sentido, Marx señala ${ }^{5}$ que la diferencia entre la mercancía que, según Smith, vende el trabajador y las demás es puramente formal.

- Historia crítica de la teoría de la plusvalia, México, Fondo de Cultura Económica, t. II, Sección 1, Cap. 3. 
El problema, así expuesto, admite dos posibles soluciones. Primera: las mercancías que son cristalización de trabajo tienen un valor según la cantidad de trabajo contenida en ellas, igual a la cantidad que pueden comprar y, el trabajo vivo, a su vez, tiene un valor según la cantidad de trabajo que pueden comprar. Esta solución tiene, entre otros, el defecto de postular dos determinaciones del valor sin señalar diferencias sustantivas entre los dos tipos correspondientes de mercancías. Segunda solución: el valor de toda mercancía es igual a la cantidad de trabajo que puede comprar. Cierto que aqui se borran diferencias no justificadas, pero el valor queda sin explicación. ¿A qué se debe que una mercancía cualquiera, trabajo cristalizado o trabajo vivo, pueda comprar la cantidad de trabajo que su valor define?

En suma: Smith señala que la ganancia es trabajo no retribuido; pero no lo explica. No logra encuadrar este hecho en una teoría del valor que abarque a todas las mercancías y no se reduzca a un círculo vicioso.

Volvamos a Ricardo. ¿Cómo explica la posibilidad de trabajo no retribuido en el contexto ilustrado por el esquema algebraico, o sea cuando el capital se limita a salarios?

En estas condiciones, es decir, cuando desaparece lo que el autor toma como capital fijo (porque no hay bienes de producción durables y todas las mercancías tienen igual ciclo de producción), el valor de cambio se determina por la cantidad de trabajo empleado en la producción antes y después de que el producto deje de pertenecer al trabajador. La cantidad relativa de trabajo cristalizada en las mercancías es independiente de la parte de ella que corresponda a los trabajadores. Por consiguiente, si la cantidad relativa de trabajo es la medida del valor de cambio antes de aparecer el trabajo asalariado, no tiene por qué no serlo después."

El valor del trabajo se determina por la cantidad de trabajo contenida en lo que Ricardo llama precio natural del trabajo: la cantidad de bienes necesaria para que la masa obrera pueda perpetuarse, sin aumentar o disminuir. ¿Con arreglo a qué normas se determina un salario que se limita a conservar la masa de obreros? ¿Qué ofrece el trabajador a cambio de la cantidad de trabajo contenido en mercancías que sólo le permiten subsistir como tal?

Esta definición del valor del trabajo no va muy lejos. Decir que el valor del trabajo es la cantidad de trabajo necesaria para producir las mercancías o el dinero que representa el salario real, equivale a señalar, en términos generales, que el valor de cualquier mercancía equivale, no al trabajo que ella contiene, sino al trabajo que contiene la mercancía o el dinero que se entrega a cambio. $\mathrm{O}$ bien hay que concluir que el valor de la mercancía que el obrero entrega al capitalista se determina por la cantidad de trabajo de que puede disponer, por estar cristalizado en el dinero o en las mercancías recibidas como salario, y que el valor de las demás mercancías se determina, en cambio, por la cantidad de trabajo que contienen. Ambas soluciones presentan los mismos escollos que las que se desprenden de Smith. En particular, ninguna aclara por qué la 
mercancía vendida por el obrero puede disponer del trabajo que supuestamente define su valor.

$\mathrm{Si}$, por otra parte, Ricardo se atiene a que el valor de todas las mercancías se determina por la cantidad de trabajo que contienen y señala que la mercancía vendida por el obrero es trabajo, o sea que el obrero entrega trabajo a cambio del que recibe cristalizado en el salario, ¿cómo concluir que la ganancia, al menos la ganancia por capital-salarios, sea la diferencia entre el valor del producto y el valor del salario?

En los Principios, lo mismo en el capítulo sobre el valor que en los capítulos sobre el salario y la ganancia, Ricardo señala que la ganancia derivada del capital-salarios proviene del hecho de que la jornada total de trabajo es mayor que la jornada para producir el salario, pero no logra hacer esto compatible con el principio de que las mercancías se cambian por el trabajo que contienen. ¿Qué vende el obrero? No puede ser la jornada, a menos que el obrero y el capitalista no intercambien mercancías equivalentes. $\mathrm{Y}$ si no son, ¿mediantę qué normas sociales podría el capitalista ejercer semejante coacción?

Tales son las preguntas que se desprenden del análisis de Marx sobre la teoría ricardiana de la ganancia, tan minuciosamente expuesto en la Historia critica de la teoría de la plusvalía. ${ }^{6}$ Ricardo señala acertadamente que la cantidad de trabajo contenido en las mercancías es independiente de su distribución entre capitalista y trabajador, pero no resuelve el dilema que formula Smith al tratar de determinar el valor de las mercancías que el trabajador vende al capitalista.

\section{CONCEPCIÓN DE MARX SOBRe EL VALOR Y TEORÍA DE LA PLUSVALÍA}

El trabajador no puede vender trabajo al capitalista porque el trabajo no es mercancía. Mercancía es cristalización de trabajo bajo la forma de valor:

Para Marx, el valor no es, como para los clásicos, un concepto con el que se trate de fundamentar las relaciones de cambio de las mercancías, sin explicar la organización de la producción en que el cambio se basa. El valor es, precisamente, la categoría que refleja el rasgo característico de la producción de mercancías: la contradicción entre el carácter social del trabajo, por obra de la división social de éste, y el carácter privado de la apropiación del trabajo.

Esta concepción del valor difiere de manera sustancial de la de Smith, e incluso de la de Ricardo. Ninguno de los dos explica por qué el trabajo es el determinante del valor. Asocian el desarrollo de la producción para el cambio a la división del trabajo. Marx, por el contrario, pone en primer término la propiedad privada sobre el producto del trabajo. Cuando el

${ }^{6}$ T. II, Sección 1, Caps, 2 y 3. 
producto es propiedad privada del productor, es el trabajo el elemento del producto que, por primera vez, se apropia el productor. Pero no por ello el trabajo deja de ser social. Hay que distribuirlo. La única forma de hacerlo es cambiándolo. He ahí por qué es el trabajo lo que se manifiesta bajo la forma valor. Los clásicos no acertaron a explicar esto. No definieron cabalmente el carácter abstracto del trabajo bajo la forma de valor.

Cuando los productores son propietarios de su producto se enfrentan unos a otros en posición de igualdad. Para intercambiar sus mercancías, deben equipararlas. Como propietarios del trabajo cristalizado en el producto, sólo pueden intercambiarlo equiparándolo a los demás trabajos. En la producción mercantil simple, como en todo modo de producción para el cambio, el trabajo sólo manifiesta su carácter social en cuanto a trabajo abstracto. El trabajo cristalizado en una mercancía es trabajo social si es equiparable al que contienen las demás. Esta cualidad del trabajo no se manifiesta directamente sino de modo indirecto al comparar las mercancías como valores.

Una mercancía es un valor de uso, pero no para su productor, sino para la sociedad. Sin embargo, la sociedad sólo puede emplearla como valor de uso si la mercancía se realiza como valor. Dicho de otro modo: si hay división del trabajo, el trabajo concreto crea valores de uso social, pero en la producción mercantil el trabajo concreto es objeto de propiedad privada. La única forma de realizarlo como creador de valores de uso social es traduciéndolo a trabajo abstracto.

En todo modo de producción para el cambio, el carácter social del trabajo sólo se manifiesta en el valor de cambio de las mercancías, desde la forma simple hasta la forma dinero. Valor no es trabajo. Valor es materialización de trabajo abstracto que únicamente se revela como valor de cambio. Es la categoría que define el enfrentamiento de propietarios de mercancías, portadoras de trabajo social.

El trabajo asalariado y su contrapartida, el capital, constituyen una relación de producción que madura con el desarrollo del cambio y que se apoya en éste. El trabajador y el capitalista se enfrentan como poseedores de mercancías. El capitalismo es un modo particular de producción de mercancías. Se explica a partir de la producción mercantil simple. La propiedad privada sobre el trabajo se desarrolla hasta el punto en que la fuerza de trabajo es objeto de propiedad privada enajenable. La posibilidad de que la fuerza de trabajo se convierta en mercancía se da con el desarrollo de la propiedad privada sobre el trabajo cristalizado en el producto, pero la necesidad de tal transformación aparece con la acumulación original del capital.

La concepción del capitalismo como modo de producción para el cambio, que se desarrolla a partir de la producción mercantil simple, implica que la categoría valor es una premisa en el análisis de la relación entre trabaio asalariado y capital. El valor es una premisa de la plusvalía.

En la producción mercantil simple no hay explotación del trabajo. 
Ningún productor puede apropiarse del trabajo de otro sin dar a cambio lo que la sociedad considera como equivalente. Al vender su mercancía, el productor recibe el equivalente en dinero de todo el trabajo directa. mente empleado en producirla y del que contienen los medios utilizados en la producción. ¿Cómo puede este sistema dar pie a la explotación del trabajo? Si los propietarios de mercancías las cambian por la cantidad de trabajo contenida en ellas, la explotación del trabajo sólo es posible si aparece en el mercado una mercancía cuyo uso suministre a quien la compra más valor del que encierra. Si es capaz de convertirse en más trabajo (abstracto) del que contiene.

La distinción de Marx entre trabajo y fuerza de trabajo es fundamental. La fuerza de trabajo, como todas las mercancías, es valor porque es cristalización de trabajo, pero, a diferencia de ellas, crea valor porque su desgaste en la producción es trabajo. Trabajo vivo; no cristalizado antes en ningún producto.

Ricardo no podía formular el problema en estos términos, porque no percibió con claridad la naturaleza del valor. El trabajador y el capitalista se enfrentan como poseedores de mercancías. El trabajador no puede vender trabajo vivo, porque ninguna mercancía es trabajo vivo. El trabajador vende una mercancía que, como todas, es cristalización del trabajo empleado en producirla. Vende fuerza de trabajo producida. Al empleársela en la producción, crea valor porque su desgaste no es más que trabajo vivo. Trabajo no cristalizado antes en ningún producto. Ricardo no advierte siquiera la diferencia entre ser valor y crear valor, puesto que no repara en la importancia entre el trabajo vivo y el trabajo cristalizado.

Los medios de producción, en cuanto tales, como valores de uso que, al aplicarles trabajo concreto, se transforman en otros valores de uso (medios de producción o de consumo) sólo puede incorporar al producto las mismas horas de trabajo abstracto que contienen. Su desgaste no es trabajo vivo.

Una hora-máquina o una hora-hectárea sólo pueden incorporar al producto la cantidad de trabajo contenido en la máquina o en la tierra. Más concretamente, la parte de esa cantidad que corresponda al desgaste de la máquina o de la tierra -como valores de uso- durante una hora.

Por el contrario, el funcionamiento de la fuerza de trabajo es trabajo vivo y como tal cualitativamente distinto del trabajo cristalizado en ella. El trabajo abstracto a que equivale el desgaste de la fuerza de trabajo es distinto al que se cristalizó en la producción de la fuerza de trabajo.

La fuerza de trabajo, como mercancía que crea valor, es la única capaz de valorizarse. De crear un valor superior al que tiene. En cualquier modo de producción la fuerza de trabajo y los medios de producción crean valores de uso. En la producción mercantil simple, la fuerza de trabajo crea valor al plasmarse en el producto como trabajo. En el capitalismo la fuerza de trabajo convertida en valor y con capacidad de crear valor, tiene la posibilidad de valorizarse. 
En El Capital ${ }^{7}$ y en los Elementos fundamentales para la crítica de la economía (Grundrisse) ${ }^{8}$ Marx explica con claridad lo específico del capitalismo como modo de producción para el cambio. $\mathrm{O}$ sea el proceso de valorización del capital invertido en fuerza de trabajo.

El divorcio entre la fuerza de trabajo y los medios de producción es condición necesaria de la existencia del trabajo asalariado y el capital, pero en la fase de producción el trabajo vivo que emana de la fuerza de trabajo se une de manera indisoluble a dichos medios. El capitalista compra fuerza de trabajo para ser utilizada durante determinado tiempo y a él pertenece el valor de uso correspondiente.

El valor de uso de la fuerza es trabajo vivo. Con esa propiedad crea valor y conserva el valor de los medios de producción. El trabajo vivo mantiene el valor de uso de los medios de producción y con ello conserva el valor de éstos. No crea su valor, porque al ser utilizados ya son trabajo materializado y subsisten como tales, aunque bajo otro forma. Al mismo tiempo, el trabajo vivo crea valor al cristalizarse como trabajo abstracto en el producto.

El capitalista no paga el valor de uso de la fuerza de trabajo. Como todo comprador de mercancías paga el valor y adquiere gratis el valor de uso. Al pagar el valor, el capitalista adquiere el valor que crea la fuerza de trabajo y la posibilidad de conservar el valor de los medios de producción. Por tanto, la diferencia entre el valor nuevo que representa el trabajo vivo y el valor de la fuerza de trabajo es trabajo que el capitalista se apropia gratis.

La clave del fenómeno está en la separación entre la fuerza de trabajo y los medios de producción. En la transformación de la fuerza de trabajo en mercancía, al trabajador, como vendedor de la fuerza de trabajo, no le corresponde ni le interesa el valor de uso de ésta, sino su valor. El valor de uso se revela en el acto de producción y, por consiguiente, es ya un momento del capital. En este sentido, la fuerza de trabajo responde al mismo mecanismo que las demás mercancías. Su valor interesa a su propietario y su valor de uso a quien la utiliza. Lo peculiar de la fuerza de trabajo es la naturaleza de su valor de uso. Si nos limitamos, como lo hemos hecho en este ensayo, a la teoría de la plusvalía, el razonamiento de Marx es bastante claro.

El análisis de todo sistema orientado al cambio debe partir de las relaciones sociales que hacen posible la creación de productos con valor de cambio. En este sentido, lo característico del sistema es la unidad dialéctica entre el carácter social del trabajo y el carácter privado de la propiedad sobre el trabajo. De ahí que el trabajo sea la sustancia que determina el valor de cambio.

Valor es lo que está detrás de valor de cambio. Trabajo abstracto cristalizado en mercancías, no trabajo vivo. El trabajo vivo tiene la po-

7 México, Fondo de Cultura Económica, 1959, t. I, Cap. V.

8 México, Siglo xxi Editores, $t$. I, pp. 299-311. 
sibilidad de convertirse en valor pero no es, en sí, valor. Por ello, en un sistema de producción mercantil, la fuerza de trabajo, el elemento de producción cuyo funcionamiento es trabajo vivo, tiene la facultad única de crear valor. La circulación de mercancías no puede crear valor. Tampoco la producción a menos que se emplee fuerza de trabajo.

El capitalismo es un modo de producción para el cambio. La explicación de la explotación del trabajo en este sistema debe partir, por consiguiente, de la ley del valor. Capitalista y trabajador asalariado se enfrentan como poseedores de mercancías y las cambian por la cantidad de trabajo abstracto que éstas tienen cristalizada. El trabajador no vende la cantidad de trabajo abstracto resultante de la jornada de producción. La duración de la jornada y el tiempo de trabajo abstracto en que se traduce, no son los elementos que evalúan la mercancía que vende el trabajador. Sólo cuenta el trabajo abstracto ya cristalizado en la fuerza de trabajo antes de que se utilice en la producción. Es decir, al determinar el valor de cambio de la fuerza de trabajo, como el de cualquier otra mercancía, lo que importa no es su valor de uso sino el trabajo empleado en su producción.

El capitalista que compra la fuerza de trabajo por su valor, se apropia del valor de uso. Esta operación tampoco es peculiar de dicha mercancía. El comprador de cualquier mercancía adquiere el valor de uso de ella al pagarla por su valor. Al vender no le interesa el valor de uso.

La fuerza de trabajo crea valor en todo sistema de producción para el cambio, pero sólo en el capitalismo es factible comparar el valor que crea con el que tiene. Ưnicamente en este sistema existe la posibilidad de valorización del capital invertido en fuerza de trabajo.

La concepción de Marx sobre las relaciones sociales de la producción para el cambio y, como modo particular de ella, del capitalismo, le llevan a una teoría de la explotación del trabajo asalariado a partir de una teoría del valor. Smith y Ricardo postulan que hay trabajo no retribuido pero no logran encuadrar el fenómeno en una teoría consistente del valor. 